\title{
Proposed Algorithm for Edge Detection in Biomedical Images based Numerical Approach
}

\author{
Ahmed Elaraby, \\ M. Hassaballah, A. S. Abdel Rady \\ Department of Mathematics, \\ Faculty of Science, \\ South Valley University, \\ Qena 83523, Egypt
}

\author{
El-Owny, Hassan Badry \\ M.A. \\ ${ }^{1}$ Department of Mathematics, \\ Faculty of Science, \\ Aswan University, \\ Aswan 81528, Egypt \\ ${ }^{2} \mathrm{CS}$ department, Taif University, \\ 21974 Taif, KSA
}

\author{
M. Heshmat \\ Department of Mathematics, \\ Faculty of Science, \\ Sohag University, \\ Sohag 81528, Egypt,
}

\begin{abstract}
In this paper we present a new algorithm for detection the edge in biomedical images depends on least square approximation method which considered one of the types of the numerical methods. Biomedical image processing has been undergoing rapid changes driven by better hardware and software. Much of the software is based on new methods. It is useful to base biomedical engineering principles on rigorous mathematical foundations for the development of software methods to be integrated into therapy systems. We focus edge detection task in biomedical images which is a key problem. We will show how proposed method can detection on the edge in images with high efficiency compare with standard methods.
\end{abstract}

\section{General Terms}

Biomedical Informatics, Image Processing.

\section{Keywords}

Edge Detection, Biomedical Images, Least Square Approximation.

\section{INTRODUCTION}

Biomedical imaging has been undergoing a revolution in the past decade with the advent of faster, more accurate, and less invasive devices. This has driven the need for corresponding software development which in turn has provided a major impetus for new algorithms in signal and image processing. Mathematical models are the foundation of biomedical computing. Basing those models on data extracted from images continues to be a fundamental technique for achieving scientific progress in experimental, clinical, biomedical, and behavioral research. Today, biomedical images are acquired by a range of techniques across all biological scales, which go far beyond the visible light photographs and microscope images of the early 20th century. Modern medical images may be considered to be geometrically arranged arrays of data samples which quantify such diverse physical phenomena as the time variation of hemoglobin deoxygenating during neuronal metabolism, or the diffusion of water molecules through and within tissue. The broadening scope of imaging as a way to organize our observations of the biophysical world has led to a dramatic increase in our ability to apply new processing techniques and to combine multiple channels of data into sophisticated and complex mathematical models of physiological function and dysfunction. A key research area is the formulation of biomedical engineering principles based on rigorous mathematical foundations in order to develop general-purpose software methods that can be integrated into complete therapy delivery systems. The microscopes are often integrated with a digital camera, which offers a possibility to use digital images. By using methods of digital image processing, the researchers in a biological field can be handled the amount of data. For that reason a need for fast and accurate methods to analyze biological data is increasing. In this work we focus edge detection task in Biomedical imaging which is a key problem.

Edge detection can defined as the process of localizing pixel intensity transitions. Once edges of an object are detected other processing such as object recognition, target tracking, segmentation, can take place $[2,3,5,6,8,9,10,11]$. In this work a novel approach based on least square approximation proposed to approximate the image data to the Gaussian function. Smoothing process used to remove noise from the image using LOG operator that represented the stander operators. It shows that the proposed approach is capable of detecting edges with high quality compared with standard methods. This paper is organized as follows: Section 2 presents some fundamental concepts of LOG filter. The proposed algorithm of edge detection is described in Section 3. Section 4 presents the effectiveness of our method when applied to biomedical image, and compare results of our algorithm against other methods. At last conclusion of this paper will be drawn in Section 5 .

\section{IMAGE FILTER}

In proposed algorithm, we use LoG operator to filter the image. The Laplacian is a 2-D isotropic measure of the 2nd spatial derivative of an image. The Laplacian of an image highlights regions of rapid intensity change and is therefore often used for edge detection. The Laplacian is often applied to an image that has first been smoothed with something approximating a Gaussian smoothing filter in order to reduce its sensitivity to noise, and hence the two variants will be described together here. The operator normally takes a single gray level image as input and produces another gray level image as output [1].

The Laplacian $\mathrm{L}(\mathrm{x}, \mathrm{y})$ of an image with pixel intensity values $\mathrm{I}(\mathrm{x}, \mathrm{y})$ is given by:

$$
\mathrm{L}(\mathrm{x}, \mathrm{y})=\frac{\partial^{2} \mathrm{I}}{\partial \mathrm{x}^{2}}+\frac{\partial^{2} \mathrm{I}}{\partial \mathrm{y}^{2}}
$$

Since the input image is represented as a set of discrete pixels, we have to find a discrete convolution kernel that can approximate the second derivatives in the definition of the Laplacian. Two commonly used small kernels are shown in Fig. 1. 


\begin{tabular}{|c|c|c|}
\hline 0 & -1 & 0 \\
\hline-1 & 4 & -1 \\
\hline 0 & -1 & 0 \\
\hline
\end{tabular}$\quad$\begin{tabular}{|c|c|c|c|}
\cline { 3 - 5 } & -1 & -1 & -1 \\
\cline { 2 - 5 } & -1 & 8 & -1 \\
\cline { 2 - 5 } & -1 & -1 \\
\hline
\end{tabular}

Fig.1: Two commonly used discrete approximations to the Laplacian filter

Using one of these kernels, the Laplacian can be calculated using standard convolution methods. Because these kernels are approximating a second derivative measurement on the image, they are very sensitive to noise. To counter this, the image is often Gaussian smoothed before applying the Laplacian filter. This pre-processing step reduces the high frequency noise components prior to the differentiation step.

In fact, since the convolution operation is associative, we can convolve the Gaussian smoothing filter with the Laplacian filter first of all, and then convolve this hybrid filter with the image to achieve the required result. Doing things this way has two advantages:

- $\quad$ Since both the Gaussian and the Laplacian kernels are usually much smaller than the image, this method usually requires far fewer arithmetic operations.

- The LoG ( Laplacian of Gaussian ) kernel can be precalculated in advance so only one convolution needs to be performed at run-time on the image.

The 2-D LoG function centered on zero and with Gaussian standard deviation $\sigma$ has the form:

$$
\operatorname{LoG}(\mathrm{x}, \mathrm{y})=-\frac{1}{\pi \sigma^{4}}\left[1-\frac{\mathrm{x}^{2}+\mathrm{y}^{2}}{2 \sigma^{2}}\right] e^{-\frac{x^{2}+y^{2}}{2 \sigma^{2}}}
$$

and is shown in Fig. 2.

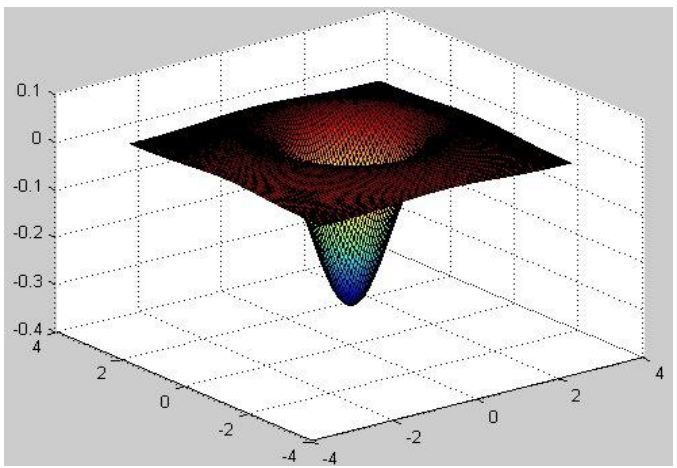

Fig. 2: The 2-D Laplacian of Gaussian (LoG) function ( $\sigma)$.

\section{PROPOSED METHOD}

In this section, we suggest a method of edge detection depends on least square carve fitting. We can represent image areas data with non-linear functions such as Gaussian Functions $[4,7]$ which its general form as :

$\gamma=\alpha \mathrm{e}^{\beta \mathrm{x}^{2}}$

This can done by applying the mask on the image plane and extract the values from the image, then rearrange the mask value ascending, So we will get data as form a pair $[\mathrm{x}, \gamma(\mathrm{x})]$, where (x) represents the location (index) of the image levels values $\gamma(x),\{x=1,2,3 \ldots n\}, n$ is the total number of pixels in the mask. After that we Re-ascending the Odd values and Re-Descending the Even values, On this basis, we can calculate the best approximation of the data using the least squares method to approximate Gaussian function to a linear function by the following steps:

By transform the equation (1) to linear form as: $\ln \gamma=\ln \alpha+\beta x^{2}$

Let $\gamma_{1}=\ln \gamma, \quad \alpha_{1}=\ln \alpha, \lambda=\mathrm{x}^{2}$, Then we can rewrite equation (2) as:

$\gamma_{1}=\alpha_{1}+\beta \lambda$

which is a linear form.

We can calculate the values of $\left(\alpha_{1}, \beta\right)$ using least square method as:

$\beta=\frac{\mathrm{n} \sum_{\lambda=1}^{\mathrm{n}} \lambda \cdot \gamma(\lambda)-\sum_{\lambda=1}^{\mathrm{n}} \mathrm{x}_{1} \sum_{\lambda=1}^{\mathrm{n}} \gamma_{1}(\lambda)}{\mathrm{n} \sum_{\lambda=1}^{\mathrm{n}} \lambda^{2}-\left(\mathrm{n} \sum_{\lambda=1}^{\mathrm{n}} \lambda\right)^{2}}$

$\alpha_{1}=\frac{\mathrm{n} \sum_{\lambda=1}^{\mathrm{n}} \gamma_{1}(\lambda)-\mathrm{b} \sum_{\lambda=1}^{\mathrm{n}} \lambda}{\mathrm{n}}$

We can calculate the value of $(\alpha)$ :

$\alpha=\mathrm{e}^{\alpha_{1}}$

After getting Gaussian relationship, we can calculate the absolute error between the values of $\gamma(\lambda)$ and $\gamma_{1}(\lambda)$ from the following equation:

error $=\frac{1}{n} \sum\left|\gamma(\lambda)-\gamma_{1}(\lambda)\right|$

We can obtain the edges in image by comparing the absolute error with the threshold value. If the absolute error value is great than threshold value, so this point represent an edge and if it less than threshold value, so this point belonging to a homogeneous area.

\section{PROPOSED ALGORITHM}

The proposed algorithm steps can be described as follows:

1. Input: A digital grayscale image I of size $\mathrm{M} \times \mathrm{N}$.

2. Applying mask on the image plane, then extract the values from the image.

3. Rearrange the mask value ascending, So we will get data as form $\gamma(\mathrm{x})$, where $(\mathrm{x}=1,2, \ldots, \mathrm{n})$.

4. Re-ascending the odd values and Re-Descending the Even values, By that we get Gaussian form.

5. Calculate the value of $(\alpha, \beta)$ from equations (4), (5) and (6).

6. Calculate the value $\gamma(\lambda)$ from equation (1).

7. Estimate the absolute error between the data $\gamma(\lambda)$ and the approximated curve $\gamma_{1}(\lambda)$, by using: (7).

8. Choose the great mean of the errors and Then compare "If error > th " then can be assigned the central mask point as $n$ edge point

9. Output: The edge detection of image I.

\section{EXPERIMENT RESULTS}

In this section, we present the performance of our proposed algorithm. "Normal Bacillus Spore" image "Fig. 3" is used to compare the results of our proposed method with the other edge detection methods under threshold value equal to 25 which can selected from experiment results." Bacillus" is a genus of bacteria of the family Bacillaceae. The genus is separated into 48 species, of which three are pathogenic. The performance of the proposed scheme is evaluated through the simulation results using MATLAB. It has been observed that the proposed algorithm works effectively compare to other methods as shown in Fig. 4. 


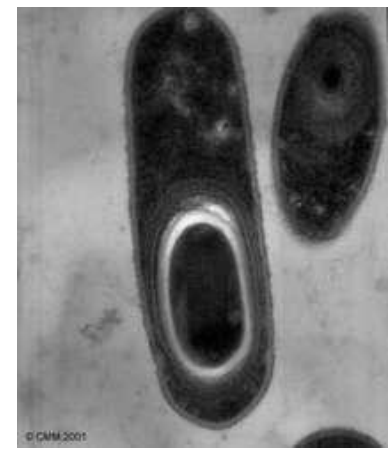

Fig.3 : Normal Bacillus Spore Image

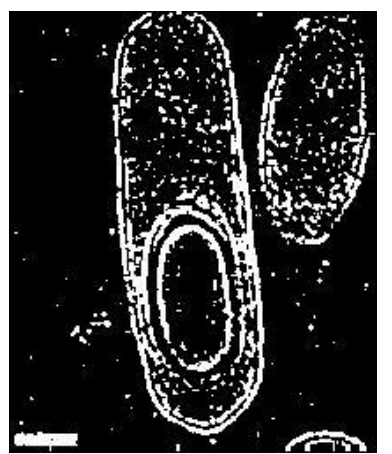

(a) : Proposed

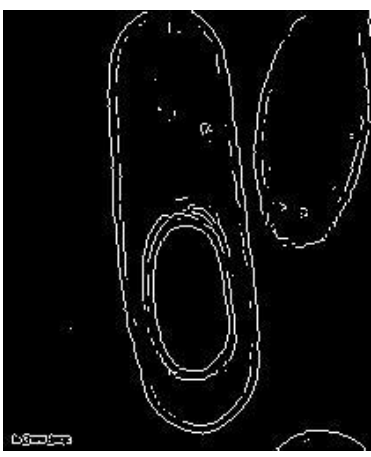

(c) : Sobel

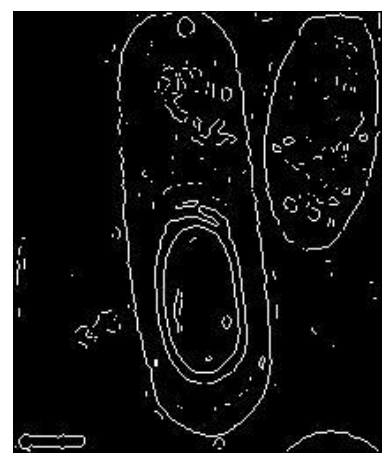

(b) : LoG

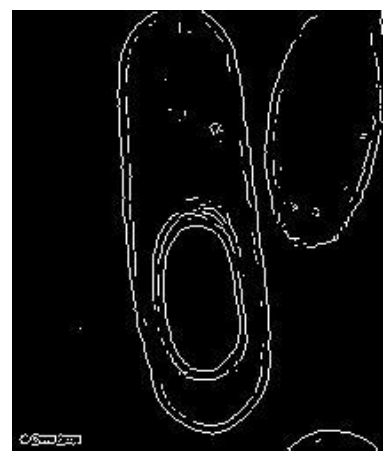

(d): Prewitt
Fig. 4: Performance of Proposed Edge Detector for "Normal Bacillus Spore"

\section{CONCLUSION}

In this paper, novel algorithm is proposed for edge detection in biomedical images based on least square approximation method which considered one of the types of the numerical methods. The traditional methods give rise to some problems like false edge detection, missing true edges, producing thin or thick lines and problems due to noise. Results demonstrate the success of the proposed method, which show that our method of achieves very encouraging results with good accuracy and simple computations. Another benefit comes from easy implementation of this method. Also our approach can extended to detection the edges of colors images directly without convert it to gray level image and this will be considered in the next article.

\section{ACKNOWLEDGMENTS}

The first author want to thankful to CAMPUS FRANCE for the financial support during his stay at Caen University, France. And he also very thankful to his colleagues at Caen University for guidelines.

\section{REFERENCES}

[1] D R. Haralick and L. Shapiro Computer and Robot Vision, Vol. 1, Addison-Wesley Publishing Company, pp $346-351,1992$.

[2] Baker S., Nayar S.K. and Murase H.," Parametric Feature Detection, Int. J.computer Vision ,vol.27, pp 27-50,1998.

[3] Gonzalez R.C. and Wintz P., "Digital Image Processing", Addision-Wesley, 1992.

[4] Richard Burden L.; Douglas Faires J.," Numerical Analysis ", Cambridge University Press, 2001.

[5] Umbough S.E., "Computer Vision and Image Processing a practical Approach Using CVIP Tools". Prentice-Hall, 1998.

[6] Yong I.T.; Gerbrands J.J.; and Van Vliet J., "Fundamentals of Image Processing", Printed in the Netherlands at the Delft University of Technology, 1998.

[7] Al-Nifaay Amer K.Hussain, Dependent The Algorithm of Linear And Gaussian Square Fitting To Determine The Edge In Digital Image “ M.sc thesis ,Babylon University College of Science, 2005.

[8] Gomes J. L. Veltto," Image processing for computer graphics", Springer-Velag, New York, Inc,1997.

[9] Gonzalez R.C. R.E. Woods and S.L. Eddins, "Digital image processing using MATLAB", Prentice Hall, 1992.

[10] Logan .S, "Developing imaging applications with XIE Lib", Prentice Hall PTR, 1998.

[11] Smith S.W, "The Scientist and Engineer's Guide to Digital Signal processing", California Technical Publishing, 1998. 